\title{
Advanced imaging and simulations of precipitate interfaces in aluminium al- loys and their role in phase transformations
}

\author{
Zezhong Zhang ${ }^{1, *}$, Laure Bourgeois ${ }^{2,3, * *}$, Yong Zhang ${ }^{3}$, Julian M. Rosalie ${ }^{4}$, and Nikhil Medhekar ${ }^{3, * * *}$ \\ ${ }^{1}$ Department of Materials Science and Engineering, Monash University, Victoria, Australia. Present address: Electron Microscopy for \\ Materials Research (EMAT), University of Antwerp, Groenenborgerlaan 171, 2020 Antwerp, Belgium; and Department of Materials, \\ University of Oxford, 16 Parks Road, Oxford OX1 3PH, United Kingdom. \\ ${ }^{2}$ Monash Centre for Electron Microscopy, Monash University, Victoria, Australia. \\ ${ }^{3}$ Department of Materials Science and Engineering, Monash University, Victoria, Australia. \\ ${ }^{4}$ Erich Schmid Institute of Materials Science, Austria. Present address: University of Leoben, Leoben, Australia.
}

\begin{abstract}
Precipitation is accompanied by the formation and migration of heterophase interfaces. Using the combined approach of advanced imaging and atomistic simulations, we studied the precipitate-matrix interfaces in various aluminium alloy systems, aiming to resolve their detailed atomic structures and illuminate their role in phase transformations.
\end{abstract}

\section{Introduction}

Being light and strong, aluminium has pinned its special position in aerospace history. From the Wright brothers' first powered flight to the current spacecraft in service, aluminium alloys are dependent on precipitation hardening for their improved strength. Once a precipitate nucleates, the heterophase interface forms as the critical boundary separating the secondary phase from the matrix; while the growth of the precipitate will be accompanied by the migration of the precipitate-matrix interfaces. Classical phase transformation theory can rationalise the critical role of interfaces in terms of thermodynamics and kinetics [1]. For instance, the interfacial energy plays an important role in the precipitate orientation relationship and the equilibrium shape, the nucleation and growth energy barrier, which ultimately governs the microstructure evolution and hardening response.

Classical analytical models assume the interface to be sharp (e.g. Becker model [2]) or diffused (CahnHilliard model [3]). However, recent microscopy studies $[4,5]$ found that precipitate interfaces may have distinct chemistry and complex intermediate structures different from the bulk phases. The associated atomic mechanisms in phase transformations remain poorly understood. First-principles methods such as density functional theory (DFT) are often used to address the atomic behaviours of precipitation, for example, Ref.[6, 7]. However, the lack of precise experimentally-based interfacial structures prevents accurate predictions even for the simplest cases. The innovation of characterisation techniques fundamentally reshaped the field of materials science. In particular, aber-

\footnotetext{
*e-mail: zezhong.zhang@uantwerpen.be

**e-mail: laure.bourgoeis@monash.edu

***e-mail: nikhil.medhekar@monash.edu
}

ration correction of electron optics significantly pushed the resolution limit of electron microscopy such that we can probe the structure, chemistry and bonding state of materials at atomic scale.

To investigate the detailed structure of precipitate interfaces, we studied the precipitate interfaces in various aluminium alloys systems using aberration-corrected scanning transmission electron microscopy. Based on the atomic structure resolved experimentally, atomistic simulations were performed to explore the underlying mechanisms. This paper will be focused on our recent progress in the textbook Al-Ag system. Together with our studies in other aluminium alloy systems, we will discuss the common observations of precipitate interfaces and their role in phase transformations.

\section{Experimental and computational methods}

\subsection{Alloy processing and sample preparation}

The Al-1.68 at.\% Ag alloy used in this work was cast from high-purity aluminium and silver. The cast ingots were homogenised at $525^{\circ} \mathrm{C}$, then hot- and cold-rolled to $0.5 \mathrm{~mm}$ alloys sheets. The samples were in the form of disks $3 \mathrm{~mm}$ in diameter and $0.5 \mathrm{~mm}$ in thickness, punched from an alloy sheet after rolling. They were solutionised at $525^{\circ} \mathrm{C}$ in a nitrate salt bath and quenched in water or oil to room temperature. This process was designed to manipulate asquenched lattice defect concentration (i.e. vacancies and dislocations), which changes the Ag clustering and following precipitation behaviours during ageing [8]. Then the samples were aged at $200^{\circ} \mathrm{C}$ in an oil bath for a range of times to characterise different stages of precipitation. The TEM specimens were made by mechanically grinding the 
disks and electro-polishing using nitric acid/methanol at $25^{\circ} \mathrm{C}$ and $13 \mathrm{~V}$ with the current around $200 \mathrm{~mA}[8,9]$. We prepared samples in other aluminium alloy systems under similar conditions.

\subsection{Electron microscopy characterisation and analysis}

The alloy microstructures and precipitate atomic structures were characterised by high-angle annular dark-field scanning transmission electron microscopy (HAADF-STEM) to exploit the large difference in the atomic numbers between the solute elements (e.g. Ag) and the $\mathrm{Al}$ matrix. High-resolution HAADF-STEM imaging was conducted in a dual-aberration-corrected FEI Titan ${ }^{3} 80$ 300 field-emission gun transmission electron microscope (FEGTEM) at $300 \mathrm{kV}$ with a $1.2 \AA$ probe and $55-200 \mathrm{mrad}$ HAADF detector. Preliminary investigations of the microstructures were carried out on a JEOL JEM 2100F FEGTEM and an FEI Tecnai $G^{2}$ F20 Super-Twin lens FEGTEM operated at $200 \mathrm{kV}$. The in situ annealing was performed on a JEOL $2100 \mathrm{~F}$ using a Gatan 625 heating holder.

To interpret the contrast of HAADF-STEM, electron microscopy images were simulated with the experimental parameters using the $\mu$ STEM software [10], implementing the multislice method with quantum excitation of phonons to incorporate elastic and inelastic scattering.

We applied statistical parameter estimation theory to locate the atomic positions at the interface, as implemented in the software StatSTEM [11]. The 3D atomic models were built by applying the known periodicity of the composing phases to the refined $2 \mathrm{D}$ positions in the viewing direction.

\subsection{Atomistic simulations}

First-principles density functional theory calculations were performed using the Vienna $A b$ initio Simulation Package (VASP) [12] with the potential constructed under the generalised gradient approximation with the treatment of Perdew, Burke, and Ernzerhof (GGA-PBE) [13]. Internal and external structural parameters were fully optimised until Hellmann-Feynman forces were less than 0.01 $\mathrm{eV} / \AA$. The convergence of the relevant energy differences concerning energy cut-off, k-point sampling and supercell size was better than $1 \mathrm{meV} /$ atom. The formation energies of different phases are given relative to the energy of FCC $\mathrm{Al}$ and $\mathrm{Ag}$ in the ground state. The atomic structures and energetics of the computed phases are given in table 1 , showing a good agreement $(<5 \mathrm{meV} /$ atom $)$ with results from Materials Genome Project [14].

The defect energy of $\mathrm{Ag}$ in solid solution was calculated by an isolated $\mathrm{Ag}$ substitutional point defect in an $\mathrm{Al}$ supercell containing 108 atoms, giving a substitutional defect energy of $0.09 \mathrm{eV}$, in reasonable agreement with previous calculations of $0.02 \mathrm{eV}$ using the local density approximation (LDA) [15]. The monolayer of Ag on $\{111\}_{\mathrm{Al}}$ plane was modelled using trigonal supercells in which the Ag monolayer was surrounded above and below by $\mathrm{Al}$ (representing the infinitely wide two-dimensionally coherent Ag plane(s) surrounded by Al matrix), containing the equivalent of 24 atomic planes of $\{111\}_{\mathrm{Al}}$. For coherent interfaces, we calculated structures with and without Ag interfacial segregation to deduce the energy difference per Ag segregated as the strain is negligible. For semi-coherent interfaces, we separated the contributions of elastic strain energy and interfacial energy by measuring the slope of formation energy against $1 / n$, where $n$ is the number of atomic planes normal to the interface.

The embedded atom method (EAM) was used to estimate the energetics of interfaces in the large systems via the Large-scale Atomic/Molecular Massively Parallel Simulator (LAMMPS) [19]. Energy and force relaxations were performed for each structure.

Understanding the atomic mechanisms of phase transformations requires identification of the local, atomiclevel structures and lattice defects. The crystal structures were recognised using adaptive common neighbour analysis [20] and dislocation analysis was performed using the dislocation extraction algorithm [21] as implemented in the Ovito software [22].

\section{Results}

The $\gamma^{\prime}\left(\mathrm{Ag}_{2} \mathrm{Al}\right)$ phase in the Al-Ag alloy system has served as a textbook example for understanding phase transformations, precipitating hexagonal close packed (HCP, space group: $P 6_{3} / m m c$ ) crystals in the face-centred cubic (FCC, space group: $F m \overline{3} m$ ) aluminium matrix. The broad facets of $\gamma^{\prime}$ precipitates are fully coherent. Distinct Ag segregation was found at the coherent interfaces of $\gamma^{\prime}$ precipitate in the early stage of aging [9, 23], as shown in Fig. 1 (a-b). In Fig. 1 (c), DFT calculations were performed to calculate the interfacial Ag segregation from the energetics perspective as a function of precipitate thickness [9]. For the semi-coherent interface, the traditionally considered atomic sharp $\{1 \overline{1} 00\}_{\gamma^{\prime}} \|\{112\}_{\mathrm{Al}}$ interface was observed to be reconstructed into a fourlayer step of $\{10 \overline{1} 1\}_{\gamma^{\prime}} \|\{111\}_{\mathrm{Al}}$ and a two-layer step of $\{10 \overline{1} 1\}_{\gamma^{\prime}} \|\{002\}_{\mathrm{Al}}$ interfaces. We explained the interfacial reconstruction using the DFT-deduced interfacial energy for each orientation relationship [9].

The edges of $\gamma^{\prime}$ precipitates are semi-coherent. According to phase transformation textbooks [1], Shockley partial dislocations are present at the semi-coherent interface to achieve the FCC-HCP structural transformation. We observed that the $\{1 \overline{1} 00\}_{\gamma^{\prime}} \|\{112\}_{\mathrm{Al}}$ interface is periodically reconstructed by nano-steps parallel to lowindex $\{002\}_{\mathrm{Al}}$ and $\{111\}_{\mathrm{Al}}$ planes, which was also seen in previous studies [23, 24]. Here we performed firstprinciples calculations to deduce the interfacial energies associated with different orientation relationships. For the atomically sharp $\{112\}_{\mathrm{Al}}$ interface, the interfacial energy was calculated to be $331 \mathrm{~mJ} / \mathrm{m}^{2}$, in good agreement with the values obtained from previous experimental estimation $\left(350 \mathrm{~mJ} / \mathrm{m}^{2}\right)$ [25] and DFT calculations $\left(325 \mathrm{~mJ} / \mathrm{m}^{2}\right)$ [18]. The interfacial energies for the $\{111\}_{\mathrm{Al}}$ and $\{002\}_{\mathrm{Al}}$ interfaces are $175 \mathrm{~mJ} / \mathrm{m}^{2}$ and $183 \mathrm{~mJ} / \mathrm{m}^{2}$ respectively. The 
Table 1. Atomic structures and energetics of face-centred cubic (FCC) and hexagonal close packed (HCP) phases in the Al-Ag alloy system. The schematic digram shows the stacking differences of close-packed planes between different phases.

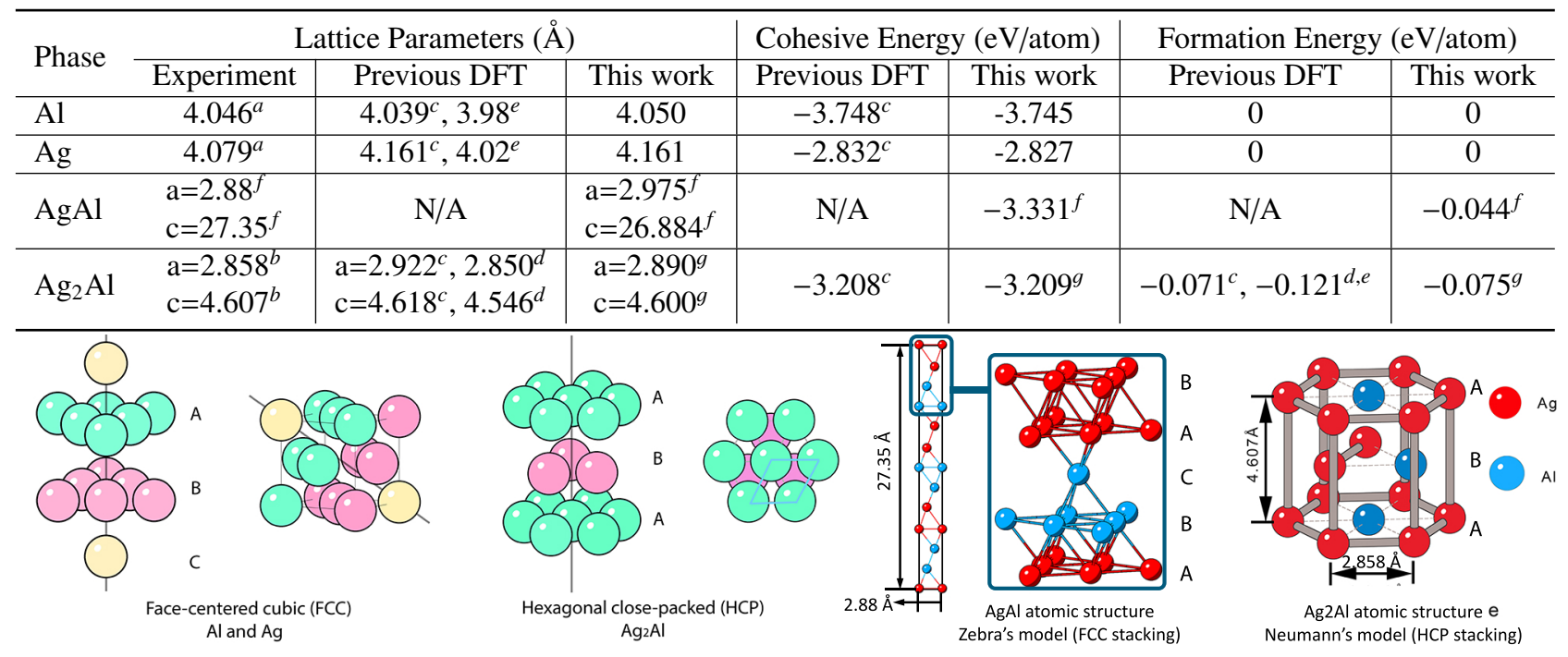

a. X-ray measurements, taken from Ref. [16].

b. X-ray measurements, taken from Ref. [17].

c. PBE-GGA calculations, taken from Materials Genome Project [14].

d. Ultrasoft-LDA calculations, taken from Ref. [6].

e. Ultrasoft-LDA calculations, taken from Ref. [18].

f. Our STEM measurements and PBE-GGA calculations, taken from Ref. [8].

g. Our PBE-GGA calculations, taken from Ref. [9].
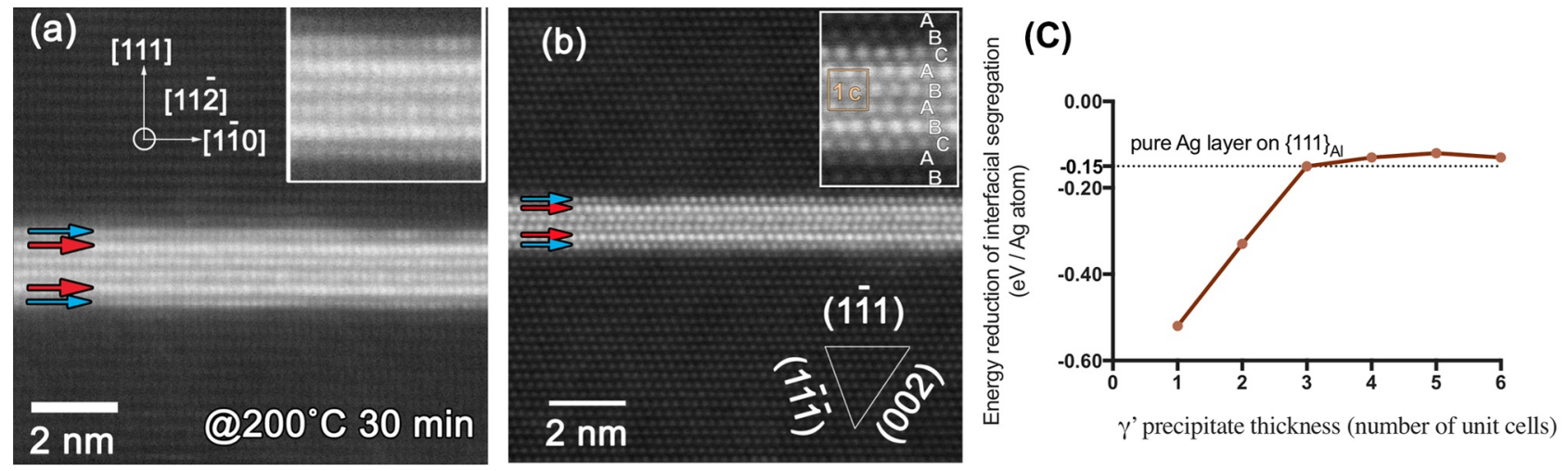

Figure 1. HAADF-STEM images of $\gamma^{\prime}$ coherent precipitate-matrix interface viewed in (a) a $\langle 112\rangle_{\mathrm{Al}}$ direction and (b) a $\langle 110\rangle_{\mathrm{Al}}$ direction, where blue arrows indicate the $\mathrm{Ag}$ segregation as reported in our previous study [23] while red arrows indicate the Ag segregation found in this study [9]. (c) Energy reduction associated with interfacial Ag segregation as a function of precipitate thickness relative to the energy state of $\mathrm{Ag}$ in solid solution. The energy of an $\mathrm{Ag}$ mono-layer on $\{111\}_{\mathrm{Al}}$ planes $(-0.15 \mathrm{eV} / \mathrm{Ag}$ atom) is displayed as a dashed line for comparison. Adapted from [9] with permission.

reconstruction can be expected to have an energetic origin: it is energetically favourable if the sum of the interfacial energies of the steps is lower than that of the sharp $\{1 \overline{1} 00\}_{\gamma^{\prime}} \|\{112\}_{\mathrm{Al}}$ interface.

Determining the exact types and locations of dislocations at the FCC-HCP interfaces is experimentally and analytically challenging. Here we combined advanced imaging with atomistic structural analysis that revealed two types of semi-coherent interfaces with unique chemical distribution and dislocation, denoted as type-I interface and Type-II interface in Fig. 2. Fig. 2(a-b) shows the HAADF-STEM image with the refined column positions as highlighted by the green and red plus signs. In the enlarged views, it is clear that the FCC/HCP transition region has a shift normal to the close-packed planes as indicated by a yellow line. By comparing the stacking of FCC (ABCABC) with that of HCP (ABABAB), we identified two layers (highlighted in red letters) that have their stacking exchanged when crossing the FCC/HCP interface. The exchanged stacking results in the atomic column in one close-packed layer being aligned on top of the next close-packed layer in the transition zone, as indicated by 


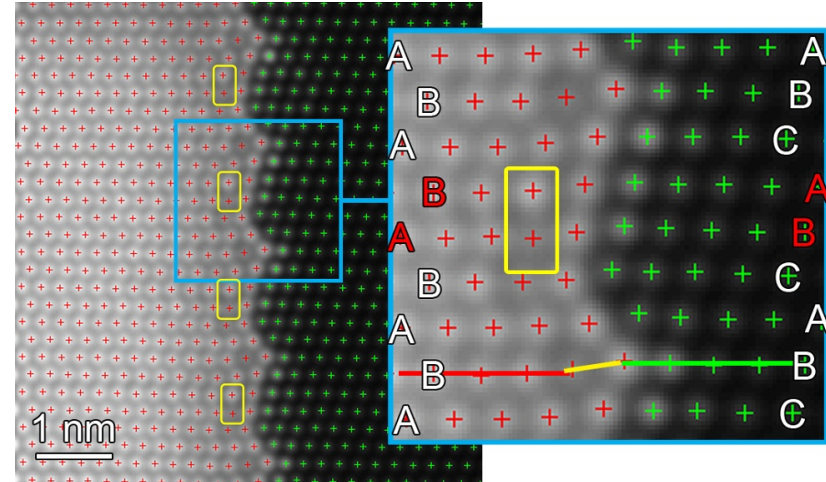

Type-I interface

shockley partial dislocations
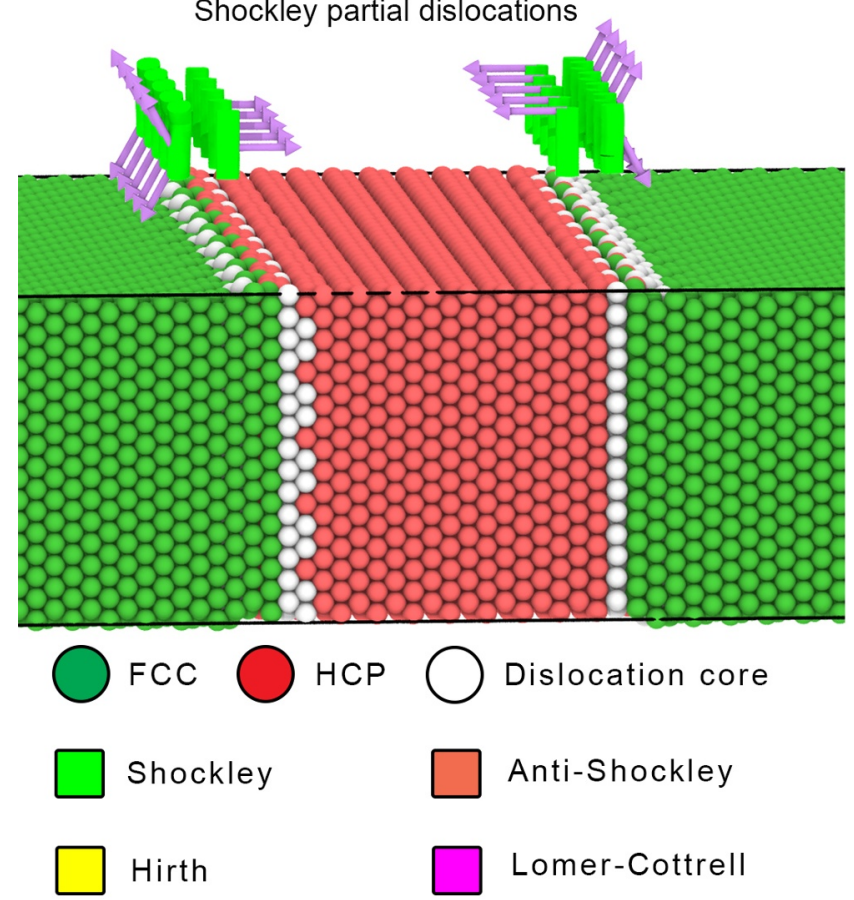

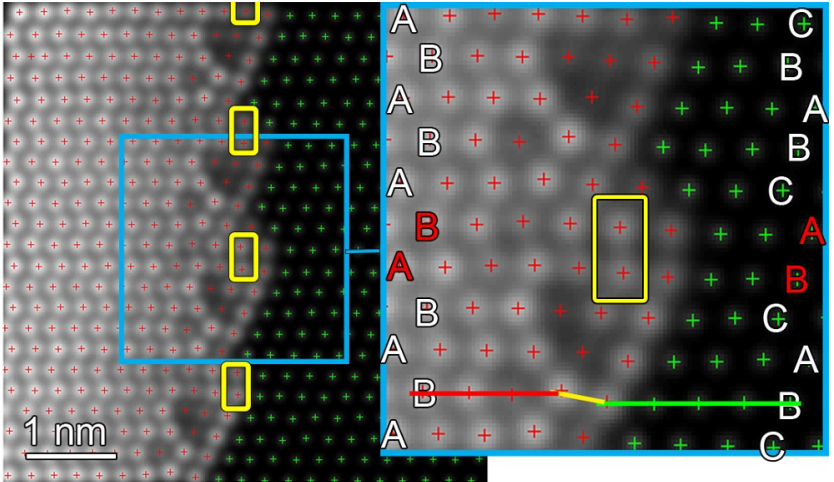

Type-II interface

Shockley partial, Lomer-Cottrell and Hirth dislocations
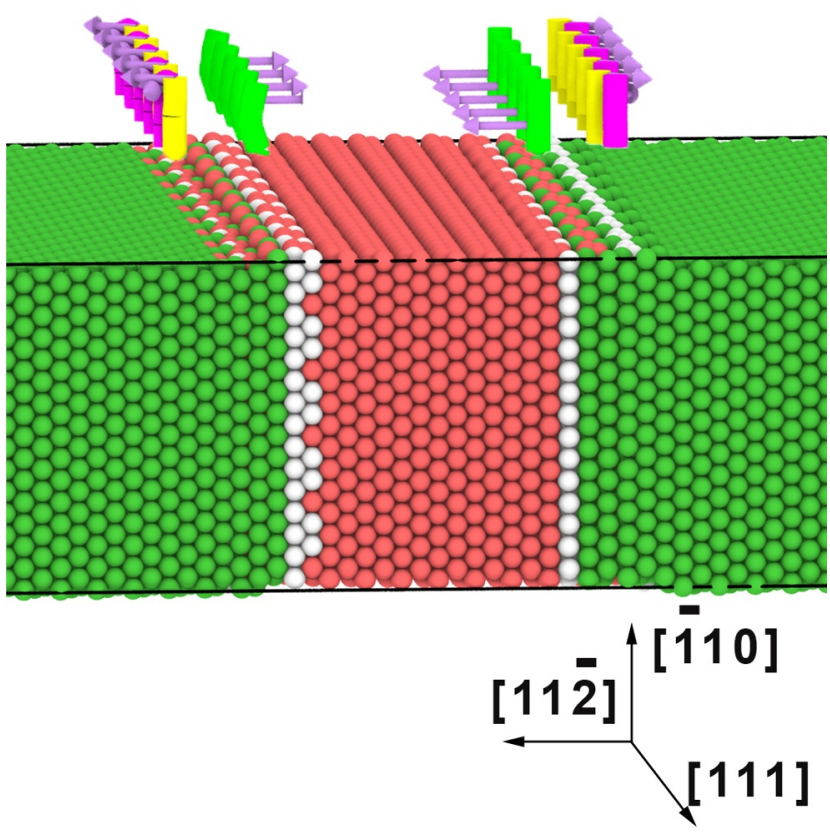

Figure 2. The coupling between chemical distributions and dislocations at semi-coherent Al- $\gamma^{\prime}$ FCC/HCP interfaces. The green and red plus signs indicate the refined atomic position via the parameter estimation theory. The yellow rectangles highlight the transition zones of the exchange of stacking from FCC (ABCABC) to HCP (ABABAB). The 3D models were built by applying the periodicity of FCC and HCP to the experimentally measured atomic positions in a $\langle 110\rangle_{\mathrm{Al}}$ direction. The adaptive common neighbour analysis was applied to determine local structure: where red atoms belong to an HCP structure, green atoms belong to an FCC structure and white atoms are inferred to be the dislocation cores. Dislocation extraction algorithm was used to determine the exact types and locations of dislocations confined in both interfaces as labelled. Reprinted from [9] with permission.

the yellow rectangular frames on HAADF-STEM images. With the precise column location obtained from imaging and known crystal periodicities of FCC/HCP in the viewing direction, we built the interfacial models for structural (using common neighbour analysis [20]) and dislocation (using dislocation extraction algorithm [21]) analysis. We should note that this 2D-to-3D model construction is only possible with interfacial edge dislocations parallel to the viewing direction. For a screw dislocation or a dislocation tilted away from the electron beam, we can no longer simply apply the crystal periodicity, which is disturbed by its strain filed. Type-I interface has three pairs of Shockley partial dislocations as expected in the textbook. Specifically, a $90^{\circ}$ Shockley partial dislocation is located in be- tween the "exchanged" layers as described above, while two $30^{\circ}$ Shockley partial dislocations are located above and below the ridges with single column Ag depletion region as shown in Fig. 2(a). Each Shockley partial dislocation is separated by two layers of close-packed planes as expected. In contrast, the Type-II interface has periodically spaced a $90^{\circ}$ Shockley partial, a Lomer-Cottrell and a Hirth dislocation. Specifically, a $90^{\circ}$ Shockley partial dislocation is still located in between the "exchanged" layers as described, while a Lomer-Cottrell dislocation is next to the three-column Ag depletion and a Hirth dislocation is next to the one-column Ag depletion as shown in Fig. 2(b). Our EAM simulations using the experimentally-informed interfacial models suggested the dislocation reaction from 
the Type-I interface to at the Type-II interface as:

$$
\begin{aligned}
& \underbrace{1 / 6[1 \overline{2} 1]+1 / 6[\overline{2} 11]}_{30^{\circ} \text { Shockley partials }} \rightarrow \underbrace{1 / 6[\overline{1} \overline{1} 2]}_{90^{\circ} \text { anti-Shockley partial }} \\
& \rightarrow \underbrace{1 / 6[\overline{1} \overline{1} 0]}_{\text {Lomer-Cottrell }}+\underbrace{1 / 3[001]}_{\text {Hirth }} .
\end{aligned}
$$

As a first-order approximation, assuming the strain energy associated with dislocations is proportional to the sum of the square of their Burgers vectors, the second part of this reaction reduces the strain energy by $50 \%$. Of course, the exact energy involves the coupling between the adjacent Shockley partial, Lomer-Cottrell and Hirth dislocations at the heterophase interface, instead of a simple superposition of their associated strain energy. We also ignored other electronic interactions due to the chemical segregation at the dislocation core, which should be included for a future study.

Despite its textbook status, the Al-Ag system has not revealed all its phase transformation secrets. For instance, we recently discovered a new phase $\zeta$ ( $\mathrm{AgAl}$, space group: $R \overline{3} m)$ in the binary system. As shown in Fig. 3(a), the $\zeta$ phase has a characteristic structure composed of alternating $\mathrm{Ag}$ and $\mathrm{Al}$-enriched bi-layers on close-packed planes, while its stacking follows that of FCC, see table 1. Fig. 3(b-e) show the time-resolved evolution of GP zone $\epsilon-\zeta-\gamma^{\prime}$ phase transformations as imaged during in situ annealing. This phase transformation pathway is triggered by lattice defects as we discovered recently for alloys heating at the nanoscale (e.g. heating the thin TEM foil), irradiation and bulk deformation $[8,26]$.

\section{Discussion}

Precipitate interfaces often have chemical segregations to lower the associated interfacial energy. In binary systems, extra $\mathrm{Cu}$ and $\mathrm{Ag}$ were found at the coherent interfaces of $\theta^{\prime}$ [4] and $\gamma^{\prime}[9,23]$ precipitates respectively. Individual Sn atoms were also observed at the $\theta^{\prime}$ semi-coherent interface [27]. Also, In-Sb nanoparticles can be attached to the coherent or semicoherent interfaces of $\theta^{\prime}$ precipitate depending on thermal history [28] and consequently change the hardening behaviour. The strength of segregation varies with precipitate thickness, which can be explained reasonably well with DFT calculations from energetics perspective. According to classical nucleation theory, interfacial energy is the critical factor that dominates the nucleation energy barrier. Thus, interfacial solute segregation is likely to be strongly favoured for precipitate nucleation.

Though Al-Ag alloys are soft and have limited engineering application, a trace amount of Ag can significantly improve the ageing response of various aluminium alloys by promoting precipitation [29, 30]. Recent studies showed Ag segregates at the precipitate matrix interfaces $[31,32]$ or participates within the precipitate phases $[33,34]$. Interestingly, the $\zeta$ phase shares structural similarities with the layered $\mathrm{Ag}$ segregation at several precipitate interfaces, including the $\theta^{\prime}$ phase in $\mathrm{Al}-\mathrm{Cu}-\mathrm{Ag}$ [35], the $\Omega$ phase in the Al-Cu-Mg-Ag [32], the $T_{1}$ phase in Al$\mathrm{Cu}-\mathrm{Li}-\mathrm{Mg}-\mathrm{Ag}$ [36] and the $\beta^{\prime \prime} / \beta^{\prime}$ phases in Al-Mg-Si-Ag [37]. The micro-alloying effect of $\mathrm{Ag}$ is expected to be deeply related to the clustering behaviour of Ag in the aluminium matrix and its interaction with defects and other solute elements.

Precipitate interfaces may develop crystallographic reconstructions and unique intermediate structures that are distinctly different from the composing bulk phases. To lower the associated interfacial energy, the high-index $\{1 \overline{1} 00\}_{\gamma^{\prime}} \|\{112\}_{\mathrm{Al}}$ semi-coherent $\gamma^{\prime}$-Al interfaces is reconstructed by $\{10 \overline{1} 1\}_{\gamma^{\prime}} \|\{111\}_{\mathrm{Al}}$ and $\{10 \overline{1} 1\}_{\gamma^{\prime}} \|\{002\}_{\mathrm{Al}}$ lowindex interfaces [9]. The reconstructed $\gamma^{\prime}$ semi-coherent interfaces have periodically spaced dislocations to achieve the FCC-HCP structural transformation. The application of atomic position refinement and atomistic analysis techniques successfully determined the types and locations of dislocations at the interfaces unambiguously. We found two types of FCC-HCP interfaces with different combinations of dislocations with unique chemical distribution at the dislocation cores as shown in Fig. 2. Further EAM simulations of the interface models showed the transformation from Type-I interface into the Type-II interface via a dislocation reaction. Previous experiments fitted the growth kinetics of $\gamma^{\prime}$ precipitate and found the thickening is inhibited [38]. Based on the in situ TEM observations, Laird and Aaronson [39] proposed a ledge mechanism in which both the coherent and the semi-coherent/incoherent interfaces of precipitates advance by ledges with different mobility. The chemical segregation and structural dislocations confined at the interfaces are expected to influence the growth kinetics of ledges, given that the Hirth and Lomer-Cottrell dislocations are know [40]. However, until now it is not clear how to calculate the interfacial mobility from the first principles. Similarly, the $\theta^{\prime}$ precipitate phase in $\mathrm{Al}-\mathrm{Cu}$ alloys has an intermediate structure composed by $\theta^{\prime \prime}$ phase and another so-called $\theta_{t}^{\prime}$ structure. Such observation may serve as the key to address the disagreement between experimental measurement and theoretical prediction of precipitate aspect ratio and growth kinetics. Future studies are needed to predict interfacial mobility based on the experimental derived atomic models.

The crystallography of the composing phases does not fully determine the structure of interfaces. For example, the $\eta\left(\mathrm{Al}_{2} \mathrm{Au}\right)$ phase and the $\theta^{\prime}\left(\mathrm{Al}_{2} \mathrm{Cu}\right)$ phase share similar crystallography but the interfacial structure is remarkably different: the coherent interface does not have extra $\mathrm{Au}$ and the semi-coherent interface is a simple combination of $\eta$ phase and the Al matrix [41]. Our DFT calculations showed that this is attributed to the much lower defect energy in aluminium of solute Au compared with solute $\mathrm{Cu}$. We found that micro-alloying of $\mathrm{Au}$ in $\mathrm{Al}-\mathrm{Cu}$ alloys can significantly improve the ageing response [42]. EDS mapping showed that Au segregates inside of the $\theta^{\prime}$ precipitates and hence promotes the $\theta^{\prime}$ nucleation $[42,43]$.

\section{Conclusion}

Since the first engineering application of aluminium alloys more than 100 years ago, their thermal and mechanical 

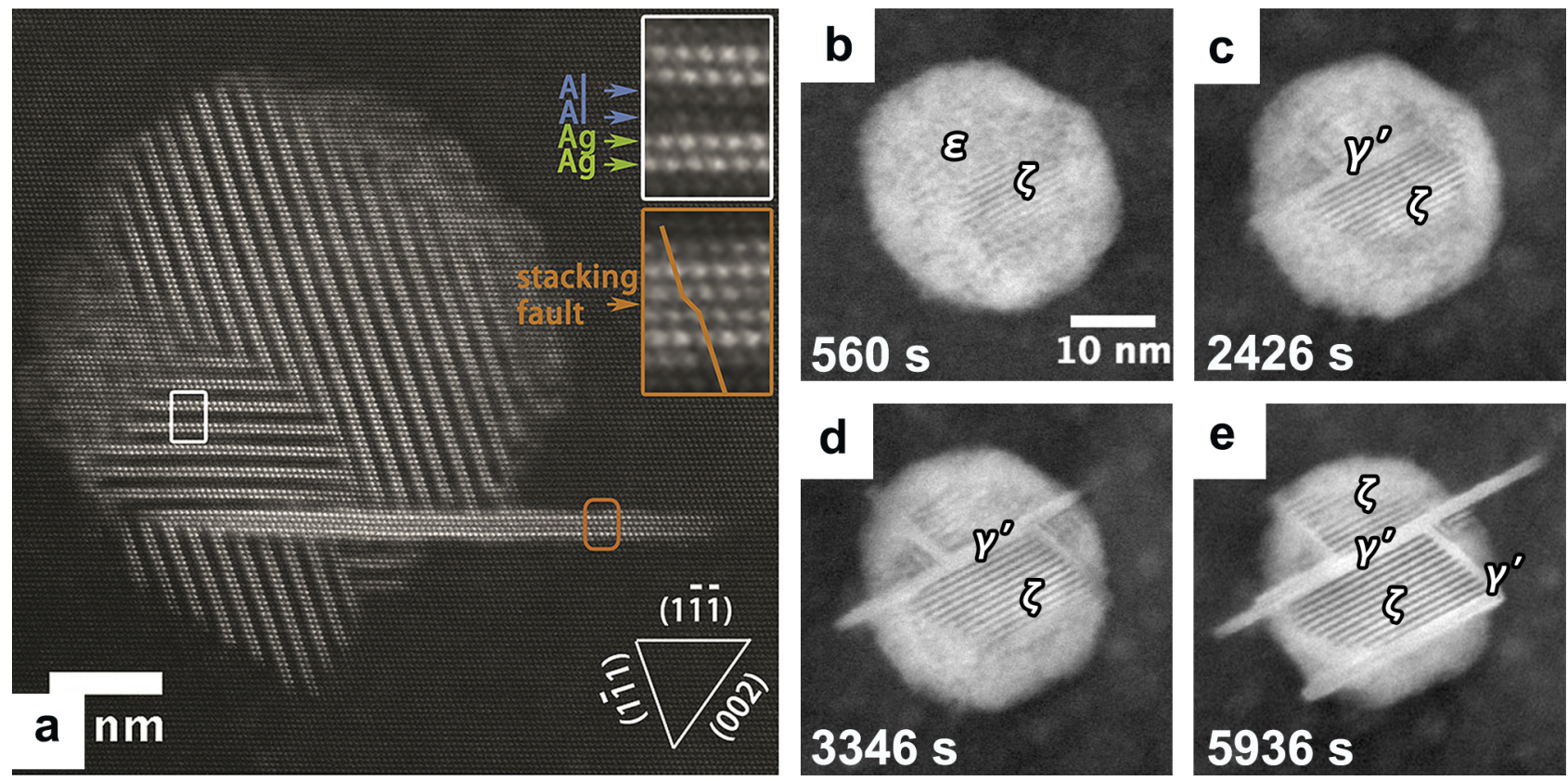

Figure 3. (a) The atomic structure of the $\zeta(\mathrm{AgAl})$ phase. The close-packed plane stacking of $\zeta$ phase follows that of FCC but with alternating Al- and Ag-enriched bilayers. (b-e) The time-resolved evolution of GP zone $\epsilon-\zeta-\gamma^{\prime}$ phase transformation as imaged during in situ annealing at $200^{\circ} \mathrm{C}$. Adapted from [8] with permission.

properties have been much improved with our knowledge of precipitation hardening. Here we inherit the insights of pioneer work by revisiting simple alloy systems using advanced imaging and atomistic simulations. A deep understanding of phase transformations requires a careful examination of the precipitate interfacial structures with aberration-corrected electron microscopy. Atomistic calculations can explain the interfacial structures reasonably well and will be increasingly important for precipitation mechanisms study at the atomic scale. Our findings show that the transformations may not be as simple as was believed, due to the intimate interplay between structure and composition confined at interfaces. With an ultimate goal of rational alloy design, further studies are needed to integrate the detailed knowledge of interfaces into the prediction of precipitate nucleation and growth kinetics.

The authors acknowledge funding from the Australian Research Council (LE0454166, LE110100223, DP150100558), computational support from Monash Advanced Research Computing Hybrid, National Computing Infrastructure and Pawsey Supercomputing Centre funded by the Australian Government, and the use of facilities within the Monash Centre for Electron Microscopy.

\section{References}

[1] H. Aaronson, M. Enomoto, J. Lee, Mechanisms of Diffusional Phase Transformations in Metals and Alloys (CRC Press, 2010), pp. 433-491, ISBN 9781439882535

[2] R. Becker, Ann. Physik 32, 128 (1938)

[3] J.W. Cahn, J.E. Hilliard, The Journal of chemical physics 28, 258 (1958)
[4] L. Bourgeois, C. Dwyer, M. Weyland, J.F. Nie, B.C. Muddle, Acta Materialia 59, 7043 (2011)

[5] L. Bourgeois, N.V. Medhekar, A.E. Smith, M. Weyland, J.F. Nie, C. Dwyer, Physical Review Letters 111, 046102 (2013)

[6] N.A. Zarkevich, D.D. Johnson, A.V. Smirnov, Acta Materialia 50, 2443 (2002)

[7] K. Kim, A. Roy, M. Gururajan, C. Wolverton, P.W. Voorhees, Acta Materialia 140, 344 (2017)

[8] Z. Zhang, L. Bourgeois, J.M. Rosalie, N.V. Medhekar, Acta Materialia 132, 525 (2017)

[9] Z. Zhang, J.M. Rosalie, N.V. Medhekar, L. Bourgeois, Acta Materialia 174, 116 (2019)

[10] L. Allen, A. D'Alfonso, S. Findlay, Ultramicroscopy 151, 11 (2015)

[11] A. De Backer, K. van den Bos, W. Van den Broek, J. Sijbers, S. Van Aert, Ultramicroscopy 171, 104 (2016)

[12] G. Kresse, J. Furthmüller, Physical Review B 54, 11169 (1996)

[13] J.P. Perdew, K. Burke, M. Ernzerhof, Physical Review Letters 77, 3865 (1996)

[14] A. Jain, S.P. Ong, G. Hautier, W. Chen, W.D. Richards, S. Dacek, S. Cholia, D. Gunter, D. Skinner, G. Ceder et al., APL Materials 1, 011002 (2013)

[15] C. Wolverton, V. Ozoliňš, Physical Review B 73, 144104 (2006)

[16] W.P. Davey, Physical Review 25, 753 (1925)

[17] J.P. Neumann, Acta Metallurgica 14, 505 (1966)

[18] D. Finkenstadt, D.D. Johnson, Physical Review B 81, 014113 (2010) 
[19] S. Plimpton, Journal of computational physics 117, 1 (1995)

[20] J.D. Honeycutt, H.C. Andersen, Journal of Physical Chemistry 91, 4950 (1987)

[21] A. Stukowski, A. Arsenlis, Modelling and Simulation in Materials Science and Engineering 20, 035012 (2012)

[22] A. Stukowski, Modelling and Simulation in Materials Science and Engineering 18, 015012 (2010)

[23] J.M. Rosalie, C. Dwyer, L. Bourgeois, Acta Materialia 69, 224 (2014)

[24] J.M. Howe, U. Dahmen, R. Gronsky, Philosophical Magazine A 56, 31 (1987)

[25] R.D. Doherty, K.E. Rajab, Acta Metallurgica 37, 2723 (1989)

[26] L. Bourgeois, Y. Zhang, Z. Zhang, Y. Chen, N.V. Medhekar, Nature communications 11, 1 (2020)

[27] L. Bourgeois, C. Dwyer, M. Weyland, J.F. Nie, B.C. Muddle, Acta materialia 60, 633 (2012)

[28] Y. Zhang, Z. Zhang, N.V. Medhekar, L. Bourgeois, Acta Materialia 141, 341 (2017)

[29] I.J. Polmear, Nature 186, 303 (1960)

[30] J.H. Auld, J.T. Vietz, I.J. Polmear, Nature 209, 703 (1966)

[31] C. Hutchinson, X. Fan, S. Pennycook, G. Shiflet, Acta Materialia 49, 2827 (2001)

[32] S.J. Kang, Y.W. Kim, J.M. Zuo, Acta Materialia 81, 501 (2014)
[33] C.D. Marioara, J. Nakamura, K. Matsuda, S.J. Andersen, R. Holmestad, T. Sato, T. Kawabata, S. Ikeno, Philosophical Magazine 92, 1149 (2012)

[34] S. Wenner, C.D. Marioara, Q.M. Ramasse, D.M. Kepaptsoglou, F.S. Hage, R. Holmestad, Scripta Materialia 74, 92 (2014)

[35] J.M. Rosalie, L. Bourgeois, Acta Materialia 60, 6033 (2012)

[36] S.J. Kang, T.H. Kim, C.W. Yang, J.I. Lee, E.S. Park, T.W. Noh, M. Kim, Scripta Materialia 109, 68 (2015)

[37] Y. Weng, L. Ding, Z. Zhang, Z. Jia, B. Wen, Y. Liu, S. Muraishi, Y. Li, Q. Liu, Acta Materialia 180, 301 (2019)

[38] M. Ferrante, R.D. Doherty, Acta Metallurgica 27, 1603 (1979)

[39] C. Laird, H. Aaronson, Acta Metallurgica Materialia 17, 505 (1969)

[40] W. Cai, W. Nix, Imperfections in Crystalline Solids, MRS-Cambridge Materials Fundamentals (Cambridge University Press, 2016), ISBN 9781107123137

[41] L. Bourgeois, Z. Zhang, J. Li, N.V. Medhekar, Acta Materialia 105, 284 (2016)

[42] Y. Chen, Z. Zhang, Z. Chen, A. Tsalanidis, M. Weyland, S. Findlay, L.J. Allen, J. Li, N.V. Medhekar, L. Bourgeois, Acta Materialia 125, 340 (2017)

[43] Y. Zheng, Y. Liu, N. Wilson, S. Liu, X. Zhao, H. Chen, J. Li, Z. Zheng, L. Bourgeois, J.F. Nie, Acta Materialia 184, 17 (2020) 